\title{
12. Bibliographie générale sur les prépositions du français
}

\section{Céline Vaguer}

\section{(2) OpenEdition}

\section{Journals}

Édition électronique

URL : https://journals.openedition.org/ml/589

DOI : $10.4000 / \mathrm{ml} .589$

ISSN : 2274-0511

Éditeur

Association Modèles linguistiques

Édition imprimée

Date de publication : 1 juillet 2006

Pagination : 171-203

\section{Référence électronique}

Céline Vaguer, «12. Bibliographie générale sur les prépositions du français », Modèles linguistiques [En ligne], 54 | 2006, mis en ligne le 01 octobre 2015, consulté le 01 juillet 2021. URL : http://

journals.openedition.org/ml/589; DOI : https://doi.org/10.4000/ml.589

Ce document a été généré automatiquement le 1 juillet 2021.

(C) Modèles Linguistiques 


\title{
12. Bibliographie générale sur les prépositions du français
}

\author{
Céline Vaguer
}

1 L'objectif est de présenter une bibliographie de travaux (livres ou articles) qui traitent des prépositions $\mathrm{du}$ français, sans limitation de point de vue (syntaxique, sémantique...), tout en essayant d'être exhaustif. La difficulté a été de savoir ce qu'il fallait retenir dans la masse disponible : faut-il se limiter au français ou étendre à l'ensemble des langues?

2 Notre choix a été, à partir de la liste des prépositions proposées par L. Mélis (2003 : 105), de " dépouiller » revues ${ }^{1}$ et recueils. Beaucoup reste à faire, certes, mais nous pensons pouvoir offrir ici un bel aperçu (1 535 références) des travaux consacrés à la préposition (première partie), aux prépositions du français (deuxième partie), et à la préposition française à travers des études contrastives (dernière partie).

\section{Recueils et articles consacrés aux prépositions du français}

3 ADLER, S. (2001) «Les locutions prépositives: questions de méthodologie et de définition », Travaux de Linguistique, 42/43 (157-170).

4 - (2002) L'ellipse des régimes des prépositions et des locutions prépositives, Thèse de doctorat, Université de Tel-Aviv.

5 - (2003) «Quand la parole est d'argent et le silence est d'or: l'ellipse comme outil argumentatif ", Revue de Sémantique et Pragmatique, 14 (81-102).

6 - (2005) « Un paramètre discursif dans l'ellipse des régimes prépositionnels », Journal of French Language Studies, 15 : 3 (219-234).

7 AMIOT, D. (2002) «Quelles relations entre les catégories de l'adverbe, de la conjonction de subordination, de la préposition et du préfixe? », Verbum, XXIV : 3 (295-308). 
8 - (2005) «Between compounding and derivation: Elements of word formation corresponding to prepositions ", in W. U. Dressler, R. Dieter R \& F. Rainer (éds) Morphology and its Demarcations, Amsterdam, John Benjamins Publishing Company (183-195).

9 - (2006) « Prépositions et préfixes », Modèles linguistiques, $53(2006,1)$.

10 AMIOT, D. \& DE MULDER, W. (2002) « De l'adverbe au préfixe en passant par la préposition : un phénomène de grammaticalisation ? ", Lingvisticae Investigationes, XXV : 2 (247-273).

11 AMIOT, D. \& MONTERMINI, F. (à par.) «Affixes et mots grammaticaux », in B. Fradin, F. Kerleroux \& M. Plénat (éds) Aperçus de morphologie du français.

12 ANSCOMBRE, J.C. (1984), «La représentation de la notion de cause dans la langue ", Cahiers de grammaire, 8 (1-53).

13 - (1986), «L'article zéro en français : un imparfait du substantif ? », Langue française, 72 (4-39).

14 - (1990), « Article zéro et structuration d'événements », in M. Charolles, S. Fischer \& J. Jayez (éds) Le discours, Presses Universitaires de Nancy (265-300).

15 - (1990), "Thème, espaces discursifs et représentation événementielle ", in J.C. Anscombre \& G. Zaccharia (éds), Milan, Unicopli (43-150).

16 - (1991a), «La détermination zéro : quelques propriétés », Langages, 102 (103-124).

17 - (1991b) «L'article zéro sous préposition », Langue française, 91 (24-39).

18 - (1994), «Proverbes et formes proverbiales : valeur évidentielle et argumentative », Langue française, 102 (95-107).

19 - (1996) «Partitif et localisation temporelle », Langue française, 109 (80-103).

20 - (1998), « Ça, c'est quelque chose. Quelques caractéristiques de la reprise d'un groupe nominal par ça/ce », Recherches en Linguistique et Psychologie cognitive, 9 (83-105).

21 - J.C. (2001), « Le rôle du lexique dans la théorie des stéréotypes », Langages, 142 (57-76).

22 - (2005), « Le ON-locuteur : une entité aux multiples visages ? », in J. Bres, P.P. Haillet, S. Mellet, H. Nolke \& L. Rosier (éds) Actes du Colloque de Cerisy Dialogisme, polyphonie: approches linguistiques', Bruxelles, De Boeck-Duculot (75-94).

23 AOKI, S. (2002), « Furansugo to nihongo tono kûkan hyôgen no taishô - 'naka' to DANS ni tsuite ", Nihongogaku, 21, Meiji Shoin, Tôkyô (74-83).

24 ASKE, J. (1989), « Path predicates in English and Spanish: A closer look », Proceedings of the Fifteenth Annual Meeting of the Berkeley Linguistics Society (1-14).

25 ASNES, M. (2006), « Le rôle des arguments datifs dans le mécanisme de la composition de l'aspect ", Revue Romane, 41 (4-20).

26 ASHER, N. \& SABLAYROLlES, P. (1994) « A typology and discourse semantics for motion verbs and spatial PPs in French », Journal of Semantics, 12: 2 (163-209).

27 ASHER, N. et col. (1995) « De l'espace-temps dans l'analyse du discours ", Sémiotiques, 9 (11-62).

28 - (1995) « Spatial, temporal and spatiotemporal locating adverbials in discourse », in P. Amsili et al. (éds) Time, Space and Movement. Meaning and Knowledge in the Sensible World, Toulouse (107-119). 
29 AUNARGUE, M. (1989) «Catégorisation des objets dans le langage: les noms de localisation interne ", Cahiers de grammaire, 14 (1-21).

30 - (1991) Contribution à l'étude de la sémantique formelle de l'espace et du raisonnement spatial : la localisation interne en français, sémantique et structures inférentielles, Thèse de doctorat d'informatique, Université Paul Sabatier, Institut de Recherche en Informatique de Toulouse.

31 - (1995) «Orientations in French spatial expressions: formal representations and inferences », Journal of Semantics, $12: 3$ (239-267).

32 - (1996), « Les noms de localisation interne - tentative de caractérisation sémantique à partir de données du basque et du français », Cahiers de lexicologie, 69 (159-192).

33 - (2001) « The Syntax and Semantics of Locating Adverbials », Cahiers de grammaire, 26 (11-35).

34 AURNAGUE, M. \& STOSIC,D. (2002), «La préposition par et l'expression du déplacement: vers une catégorisation sémantique et cognitive de la notion de «trajet » ", Cahiers de lexicologie, 81 (113-140).

35 AUNARGUE, M. \& VIEU, L. (1993) «A threelevel approach to the semantics of space ", in C. ZelinskyWibbelt (éd) The Semantics of Prepositions: From Mental Processing to Natural Language Processing, Berlin, Mouton de Gruyter (393-439).

36 AUNARGUE, M., VIEU, L. \& BORILlo, A. (1997) «Représentation formelle des concepts spatiaux dans la langue », in M. Denis (éd) Langage et cognition spatiale, Paris, Masson (69-102).

37 BAARDEWIJKRESSEGUIER, J. (van) (1983) «La nonalternance entre syntagme prépositionnel et adjectif de relation ", Cahiers de lexicologie, 43 (73-84). BADER, F. (1997) «Prépositions dans les langues indoeuropéennes anciennes », Faits de langues, 9 (49-60).

\section{français}

44 

temps ", in G. Kleiber \& J.E. Tyvaert (éds) L'anaphore et ses domaines, Paris, Klincksieck $(1-50)$.

54 - (1998) «Espace et temps : quelle place pour la métaphore », Verbum, XX : 4 (353-382).

55 - (1999), « Prendre Marie par la main / Le couteau par le manche, ou comment par joue... la partie ", in D. Amiot et alii (éds) Fonctions syntaxiques et rôles thématiques, Arras, Artois Presses Université, coll. Cahiers scientifiques de l'Université d'Artois, 13 (9-25).
V SHYLDKROT , H. \& KEMMER, S. (1988) « Le développement sémantique des conjonctions en français, quelques concepts généraux ", Revue romane, 23 : 1 (9-19).
BEKKERS, F. H. H. A. (1931) Etude sur l'emploi que Froissart fait de la Préposition, Amsterdam, H. J. Paris. Benveniste (1966) Problèmes de linguistique générale, 1, Paris, Gallimard (132-139)] Travaux de linguistique, 14/15 (67-81).

- (1989) Composantes linguistiques de la référence temporelle. Les compléments de temps, du lexique à l'énoncé, Thèse de doctorat d'Etat, Université de Paris VII.

BERTHONNEAU, A.-M. \& CADIOT, P. (éds) (1991) Langue française $\mathrm{n}^{\circ} 91$ : Prépositions, représentations, référence, Paris, Larousse.

- (1993) Lexique $\mathrm{n}^{\circ} 11$ : Les prépositions : méthodes d'analyse, Lille, Presses Universitaires de Lille.

BERTHOUD, A.C. (1996), Paroles à propos - Approche interactive et énonciative du topic, Paris, Ophrys.

BIERWISCH, M. (1988) « On the Grammar of Local Prepositions », in M. Bierwisch, W. Motsch \& I. Zimmermann (éds) Syntax, Semantik und Lexikon, Berlin, Akademie Verlag, coll. Studia Grammatica, XXIX (1-65).

BISSELL, C. H. (1944) «Repetition of prepositions with more than one subject in French », The French Review, 19 (199-203).

BLAISE, A. (1948) «L'emploi prépositionnel des substantives dans le latin postclassique ou tardif », Revue des études latines, 26 (64-65).

BLANCHE-BENVENISTE, C. (1998) «Présence et absence de prépositions dans les clivées et les pseudoclivées ", in A. Englebert et al. (éds) Actes du XXII ème Congrès International de Linguistique et de Philologie Romanes, VI, Tübingen, Niemeyer (55-65).

- (2001) « Préposition à éclipses », Travaux de Linguistique, 42/43 (83-95).

BLINKENBERG, A. (1960) Le problème de la transitivité en français moderne, Copenhague, Ejnar Munksgaard.

BOISSON, C. (1998) « Les adverbiaux sans prépositions », in S. Rémigiraud \& A. Roman (éds) Autour du circonstant, Lyon, PUL (215-259).

BONAMI, O. (1999) Les constructions du verbe: le cas des groupes prépositionnels argumentaux, Thèse de l'Université Paris VII. 
Lexique et Traitement Automatique des Langages, GRECO-GALF, Toulouse, Université Paul Sabatier (11-23).

77 - (1986) «La quantification temporelle : durée et itérativité en français », Cahiers de grammaire, 11 (117-156).

78 - (1988a) «L'expression de la durée : construction des noms et des verbes de mesure temporelle », Lingvisticae Investigationes, XII : 2 (363-396).

79 - (1988b) «Le lexique de l'espace : les noms et les adjectifs de localisation interne ", Cahiers de grammaire, 13 (1-22).

80 - (1998c), L'espace et son expression en français, Paris, Ophrys.

81 - (1990) « A propos de la localisation spatiale », Langue française, 86 (75-84).

82 - (1991) «Quelques marqueurs de la deixis spatiale », in M. A. Morel \& M. DanonBoileau (éds) La Deixis, Paris, PUF (246-255).

83 - (1992) «Le lexique de l'espace : prépositions et locutions prépositionnelles de lieu en français ", in L. Tasmowski \& A. ZribiHertz (éds) De la musique à la linguistique. Hommages à Nicolas Ruwet, Ghent, Blandijnberg 2, coll. Communications \& Cognition (176-190).

84 - (1993) «Prépositions de lieu et anaphore », Langages, 110 (27-46).

85 (1995) «Quelques schémas de syntagmes à redoublement ", in H. BatZeev Shyldkrot \& L. Kupferman (éds) Tendances récentes en linguistique française et générale, Amsterdam, Benjamins (95-109).

86 - (1996) «Le déroulement temporel et sa représentation spatiale en français », Cahiers de praxématique, 27 (109-128).

87 - (1997) « Aide à l'identification des prépositions composées de temps et de lieu », Faits de langues, 9 (175-184). 
88 - (1998a) L'espace et son expression en français, Paris, Ophrys.

89 - (1998b) «Quand le complément direct d'objet est un lieu », Travaux de linguistique, 35 (51-65).

90 - (1999a) «Les adverbes de référence temporelle comme connecteurs temporels de discours ", Bibliothèque des Cahiers de l'Institut de Linguistique de Louvain, 99 (131-145).

91 - (1999b) «Partition et localisation spatiale: les noms de localisation interne", Langages, 136 (53-75).

92 - (2000) «Degrés de grammaticalisation: des noms de parties aux prépositions spatiales ", in P. de Carvalho \& L. Labrune (éds) Grammaticalisation. 1. (Dé) motivation et contrainte, Rennes, Presses Universitaires de Rennes, coll. Travaux du Cerlico, 13 (257-274).

93 - (2001) « Il y a prépositions et prépositions », Travaux de linguistique, 42/43 (141-155).

94 - (2003), «Un ou plusieurs contre? ", dans Recherches linguistiques, 26, 2003, « CONTRE Identité sémantique et variation catégorielle », P. Péroz éd., Université de Metz (45-64).

95 - (2005) «Peuton identifier et caractériser les formes lexicales de l'aspect en français?", in H. Bat-Zeev Shyldkrot \& N. Le Querler (éds) Les périphrases verbales, Amsterdam/Philadelphia, John Benjamins, coll. Lingvistiace Investigationes : Supplementa, 25 (67-82).

BORKIN, A. (1973), "To be and not to be ", Papers from the Regional Meeting of the Linguistics Chicago Society 9 (44-56).

BOSSONG, G. (1980) «Questions de linguistique chinoise : réflexions critiques au sujet du livre de Claude Hagège, Le problème des Prépositions et la Solution Chinoise », Lingua, 51 (233-250).

BOSTRÖM, I. (1957) Les noms abstraits accompagnés d'un infinitif et combinés avec 'avoir'. Etude historique sur la syntaxe des articles et des prépositions dans ce genre de constructions françaises, Paris / Lund / Copenhague, Boyveau \& Chevillet / C. W. K. Gleerup / Ejnar Munksgaard.

BOSWELL, R. E. (1969) A semantic theory of French grammar and its application to the preposition before infinitive, Diss., University of Yale.

BOUGAULT, L. (2000) «Les prépositions dans les trois premiers poèmes de Stèles de Victor Segalen », L'Information Grammaticale, 85 (24-30).

101 BRANDT, P. A. (1971) «Pour une chromatique. Note sur la valeur des prépositions françaises », Lingua e Stile, $6: 2$ (201-205).

BRÉAL, M. (1982), Essai de sémantique, Paris, Hachette (1 ${ }^{\text {ère }}$ éd. 18-97).

BREBAN, V. (1957) «Les prépositions dans les dictionnaires unilingues », Mélanges linguistiques publiés à l'occasion du $8^{\text {ème }}$ Congrès International des Linguistes à Oslo du 5 au 9 août 1957, Bucharest, Ed. Acad. Rep. Pop. Roum. (201-205).

BRONDAL, V. (1939) «L'originalité des prépositions du français moderne », Mélanges de linguistique offerts à Charles Bally, Genève, Librairie de l'Université (337-346). [repris dans (1943) Essais de Linguistique Générale, Copenhague, Munksgaard (81-89)]

105 (1948) Les parties du discours. Partes orationis. Etudes sur les catégories linguistiques, Copenhague, Munksgaard. 

cognitifs », Cahiers de lexicologie, 58 (63-79).

113 - (1992a) "Vers une réduction cognitive de la préposition ", in D. Andler et al. (éds) Epistémologie et cognition, Liège, Editions Pierre Mardaga (239-253).

114 - (1992b) «Conditionnements et préparations culinaires : notes », in L. Tasmowski \& A. ZribiHertz (éds) De la musique a la linguistique. Hommages à Nicolas Ruwet, Ghent, Blandijnberg 2, coll. Communications \& Cognition (205-214).

115 - (1997a) Les prépositions abstraites du français, Paris, A. Colin.

116 - (1997b) «Les paramètres de la notion de préposition incolore », Faits de langues, 9 (127-134).

117 - (1999a) «Espaces et prépositions ", Revue de Sémantique et Pragmatique, 6 (43-70).

118 - (1999b) «Les sens de jouer: esquisse d'une approche par le biais de attaches prépositionnelles ", in F. Cordier \& J.E. Tyvaert (éds) Les prépositions dans la rection des verbes, Reims, Presses Universitaires de Reims, coll. Recherches en Linguistique et Psychologie cognitive, 11 (105-129).

119 - (2000) «La préposition comme connecteur et la prédication seconde", Langue française, 127 (112-125).

120 - (2001) «Eléments d'une critique de la notion de préposition spatiale», Syntaxe \& Sémantique, 3 (117-129).

121 - (2002a) «Schémas et motifs en sémantique prépositionnelle : vers une description renouvelée des prépositions dites 'spatiales' ", Travaux de linguistique, 44 (9-24).

122 - (2002b) «Schematics and motifs in the semantics of prepositions », in S. Feigenbaum \& D. Kurzon (éds) Prepositions in their Syntactic, Semantic and Pragmatic Context, Amsterdam, John Benjamins (41-57).

CADIOT, P. \& IBRAHIM, A. (éds) (1999) Revue de Sémantique et Pragmatique, nº 6 : Approches sémantiques des prépositions, Orléans, Presses Universitaires d'Orléans.

124 - (1999) «Approches sémantiques des prépositions ", Revue de Sémantique et Pragmatique, $6(9-12)$.

125 CADIOT, P. \& VISETTI, Y.M. (2001) Pour une théorie des formes sémantiques - motifs, profils, thèmes, Paris, PUF.

126 - (2002) «Instability and the theory of semantic forms: Starting from the case of prepositions ", in S. Feigenbaum \& D. Kurzon (éds) Prepositions in their Syntactic, Semantic and Pragmatic Context, Amsterdam, John Benjamins (9-39). 
CAMPRUBI, M. (2005) « Prépositions et classes ou sousclasses de prédicats et d'objets », in I. ChoiJonin et coll. (éds) Questions de classification en linguistique: méthodes et descriptions, Berne, Peter Lang, coll. Sciences pour la communication, 78 (81-98).

CAMUS, R. \& DE VOGUE, S. (éds) (2004), Linx $\mathrm{n}^{\circ} 50$ : Variation sémantique et syntaxique des unités lexicales : étude de six verbes français, Université de Paris X-Nanterre.

CARLIER, A. (2006) « From preposition to article: the grammaticalization of the French partitive », Studies in Language, 30: 4 (655-703).

CERVONI, J. (1981) " Méthodes en linguistique contrastive ", in C. Schwarze (éd) Analyse des prépositions, Tübingen, Max Niemeyer Verlag (1-18).

- (1984) «Prépositions et continuum », Modèles linguistiques, VI : 2 (87-98).

- (1988) Sémantique prépositionnelle. Essai critique sur les théories de la préposition, Thèse d'état, Paris IV.

- (1990a) «Prépositions et compléments prépositionnels », Langue française, 86 (85-89).

- (1990b) «Sémantique prépositionnelle. Essai critique sur les théories de la préposition », L'Information Grammaticale, 45 (43-44).

- (1991) La préposition : étude sémantique et pragmatique, Louvain-laNeuve/Paris, Duculot.

CHAROLLES, M. (1997) «L'encadrement du discours - Univers, champs, domaines et espaces ", Cahiers de recherche linguistique, 6 (1-73)

- (2003) «De la topicalité des adverbiaux détachés en tête de phrase", Travaux de linguistique, 47 (11-49).

CHAROLLES, M. \& VIGIER, D. (2005) «Les adverbiaux en position préverbale : portée cadrative et organisation des discours ", Langue française, 148 (9-30).

CHERVEL, A. (1979) «Rhétorique et grammaire: petite histoire du circonstanciel ", Langue française, 41 (5-19).

CHOUL, J.C. (1982) «Le comportement syntagmatique et l'évolution de quelques prépositions dans le français contemporains", Revue de l'Association Québécoise de Linguistique, $1: 1 / 2$ (35-44).

- (1982) «Le Vide prépositionnel et le processus néologique idiomatique ", Revue de l'Association Québécoise de Linguistique, $2: 2$ (21-30).

2 CHOI-JONIN, I. (2003) «Ordre syntaxique et ordre référentiel. Emplois de la locution prépositive Quant à», in B. Combettes, C. Schnedecker \& A. Theissen (éds) Ordre et distinction dans la langue et le discours, Paris, Honoré Champion (133-147).

CLEDAT, L. (1901) « La préposition et l'article partitif », Revue de Philologie Française et de Littérature, 15 (81-131).

CONFAIS, J.P. (1989) «Les notions de "circonstance» , "complément circonstanciel» et "circonstant" ", in C.R.L.G. (éd) La relative - La fonction circonstancielle, Université de Nice (103-127).

CORDIER, F. \& TYVAERT, J. E. (1999) Les langues des sourds: des prépositions dans la rection des verbes de développement du langage avant deux ans : actes des journées scientifiques $d u$ CIRLEP, Reims, Presses Universitaires de Reims.

CORTIER, C. (2001) «Les syntagmes prépositionnels prédicatifs dans les grammaires universitaires : un observatoire de la place accordée aux prépositions ", Travaux de linguistique, $42 / 43$ (121-140). 
CoX, T. J. (1983) «Teaching the Unteachable: Prepositional Complementizers in French », The French Review, Journal of the American Association of Teachers of French, 57 (168-178).

CULIOLI, A. \& DESCLES, J.P. (1981) Systèmes de représentations linguistiques et métalinguistiques: Les catégories grammaticales et le problème de la description de langues peu étudiées, coll. ERA 642, Université de Paris VII.

CUNITA, A. (2005) "Comment opère la charge sémantique de la préposition", in M. Tenchea \& A. Tihu (éds) Prépositions et conjonctions de subordination. Syntaxe et sémantique, Timisoara, Editura Excelsior Art (75-86).

CUYCKENS, H. (1984) «The deictic vs. nondeictic ambiguity in the Spatial uses of Prepositions », Lingvisticae Investigationes, VIII: 1 (187-194).

CUYCKENS, H. \& RADDEN, G. (2002) Perspectives on Prepositions, Tübingen, Niemeyer.

CULIOLI, A. (2000), Pour une linguistique de l'énonciation. Opérations et représentations, Tome 1, Gap/Paris, Ophrys (1 $1^{\text {ère }}$ éd. 19-90).

DAGNAC, A. (2005) «Description et interprétation spatiale : l'incipit des Choses de G. Perec ", in I. ChoiJonin et col. (éds) Questions de classification en linguistique: méthodes et descriptions, Berne, Peter Lang, coll. Sciences pour la communication, 78 (99-125).

DALADIER, A. (1999) «Origine adverbiale du génitif indoeuropéen, extractions, possessifs, anaphore associative et interprétations grammaticalisées dans les GN du français ", Langue française, 122 (101-125).

- (1999) « Auxiliation des noms d'action », Langages, 135 (87-107).

DAMOURETTE, J. \& PICHON, E. (1911/1940) (rééd. 1969) Des mots à la pensée. Essai de grammaire de la langue française, t. VII, chap. XVII : Prépositions, Paris, d'Artrey (227-296).

DANELL, K. J. (1974) Le groupe 'substantif + préposition + substantif en français contemporain. Etude sémantique et syntaxique, Acta Universitatis Upsaliensis, coll. Studia Romanica Upsaliensia, 15, Stockholm, Almqvist \& Wiksell.

DANLOS, L. (1980) Représentation d'informations linguistiques : les constructions 'N être Prép X', Thèse de $3^{\text {ème }}$ cycle, L.A.D.L., Université Paris VII.

- (1980) «Représentation d'informations linguistiques : les constructions N être Prép X », Lingvisticae Investigationes, IV : 2 (443-445).

- (1988) « Les phrases à verbe support être Prép », Langages, 90 (23-37).

DANNBERG, T. (1962) «L'emploi de la préposition locale devant les noms de département dans le français contemporain ", Moderna Sprâk, 56 (46-56).

DANON-BOILEAU, L. \& MOREL, M.A. (éds) (1997) Faits de langues, n 9 : La préposition: une catégorie accessoire ?, Paris, Ophrys.

DANONBOILEAU, L. \& MOREL, M.A. (1997) «Question, point de vue, genre, style... : les noms prépositionnels en français contemporain ", Faits de langues, 9 (193-200).

DEBAISIEUX, J.M. (2001) « Contraintes syntaxiques et discursives des emplois de quant à et en ce qui concerne en français parlé ", Cahiers de praxématique, 37 (125-146).

DELATTRE, P. (1964a) «Le jeu des prépositions dans l'enchaînement des verbes en français ", The French Review, $21: 2$ (2032) \& 38 (67-81). 
166 - (1964a) «French prepositional patterns in linking a verb to an infinitive object ", Modern Language Journal, 48 (29-35).

DELAUNOY, A. (1967) Le bon emploi de la préposition en français, Namur, WesmaelCharlier.

DELAVEAU, A. (1992) "Les compléments circonstanciels et l'analyse syntaxique ", Le Gré des langues, 3 (188-201).

DELAVEAU, A. (2001), Syntaxe. La phrase et la subordination, Armand Colin.

DEMAGNY, A.C. \& PAPROCKA-PIOTROWSKA, U. (2004) "L'acquisition du lexique verbal et des connecteurs temporels dans les récits de fiction en français L1 et L2 ", Langages, 155 (52-75).

171 DEMONTE, V. (1987) « Command, Prepositions, and Predication », Linguistic Inquiry, 18 (189-204).

DE MULDER, W. (2001), «Présentation : la linguistique diachronique, les études sur la grammaticalisation et la sémantique du prototype ", Langue française, 130 (8-32)

DE MULDER, W. \& FLAUX, N. (1998) Verbum, XX : 4 : Prépositions et métaphore, Nancy, Presses Universitaires de Nancy.

174 DENDALE, P. (2001), "L'emploi spatial de contre: propositions pour un traitement unifié », Travaux de Linguistique, 42/43 (229-239).

175 - (2003), «La polysémie de contre: quelques hypothèses pour lier spatial et non spatial ", in P. Péroz (éd) Contre 'Identité sémantique et variation catégorielle', Université de Metz, coll. Recherches linguistiques, 26 (65-90).

DERVILLEZ-BASTUJI, J. (1982) Structure des relations spatiales dans quelques langues naturelles - Introduction à une théorie sémantique, Paris/Genève, Droz.

177 DESCLES, J.P. (1987) «Réseaux sémantiques: la nature logique et linguistique des relateurs ", Langages, 87 (55-78).

178 - (2001a) «Prépositions spatiales, relateurs et préverbes », in A. Rousseau (éd) $L a$ sémantique des relations, Lille, Université Charles de Gaulle Lille 3 (87-99).

179 - (2001b) «Prépositions spatiales, relateurs et préverbes », Etudes cognitives, 4, Varsovie, Instytut Slawistyki, Polska Akademia nauk (13-30).

180 DESSAUX, A.M. (1976a) «Déterminants nominaux et paraphrases prépositionnelles: problèmes de description syntaxique et sémantique du lexique », Langue française, 30 (4462).

181 - (1976b), «A propos de quatre compléments formés avec par et d'un nom temporel (Ntps) : propriétés syntaxiques et signification ", in J.C. Chevalier \& M. Gross (éds) Méthodes en grammaire française, Paris, Klincksieck (41-72).

182 - (1978) «Les compléments distributifs : un sous-système formel et sémantique de la complémentation en français ", Langue française, 39 (5-29).

DESSAUX-BERTHONNEAU, A.M. (1985) « Niveaux et opérations dans la description des compléments temporels ", Langue française, 66 (20-40).

DESTUTT de TRACY, A. L. C. (1970) «Des prépositions ", Eléments d'Idéologie, Paris, Vrin, vol. 2 : La Grammaire, Chapitre III, Paragraphe V (105-120). 
DEULOFEU, J. (1982) «A propos des préjugés logicistes dans l'analyse grammaticale : le cas des prépositions; hypothèses linguistiques et sociolinguistiques ", Recherches sur le Français Parlé, 4 (45-68).

DE VOGUÉ, S. (1991), « La transitivité comme question théorique : querelle entre la théorie des positions de J.C. Milner et la théorie des opérations prédicatives et énonciatives de A. Culioli », Linx, 24 (37-65).

DE VOGUÉ, S. \& PAILlARD, D. (1997), «Identité lexicale et hétérogénéité de la variation. Le cas de suivre ", in C. Guimier (éd) Cotexte et calcul du sens, Caen, Presses Universitaires de Caen (41-61).

DÖPKE, W. \& SCHWARZE, C. (1981) «Le rôle des prépositions locales dans la constitution sémantique de la phrase ", in C. Schwarze (éd) Analyse des prépositions, Tübingen, Max Niemeyer Verlag (19-28).

DRIOTON, E. (1941) «Expressions prépositionnelles d'identité », Annales du Service des Antiquités de l'Egypte, 40 (619-621).

DRYER, M. S. (1986), « Primary Objects, Secondary Objects, and Antidatives », Language, 62 (808-845).

DUBOIS, D. (1993) «Analyse de 22 catégories sémantiques du français", L'Année psychologique, 83 (465-489).

DUBoIS-CHARLIER, F. (2001) «Compléments de Verbe, de Proposition, de Phrase, d'Énoncé », Travaux, 17 (33-50).

DUBOIS, J. \& DUBOIS-CHARLIER, F (1997), Les verbes français, Diffusion Paris, Larousse.

DUBSKY, O. (1929) Sur quelques particularités dans l'emploi du cas régime et du cas prépositionnel dans la fonction de complément adnominal, Paris, Champion.

DUCROT, O. (1984), Le dire et le dit, Paris, Minuit.

DUGAS, A. (2000a) Le verbe et ses prépositions, Montréal, Les Editions Logiques.

- (2000b) «Quelques prépositions introduisant des compléments essentiels », BULAG Lexique, Syntaxe, Sémantique. Mélanges offerts à Gaston Gross à l'occasion de son $60^{\text {ème }}$ anniversaire (183-198).

- (2001) « Une analyse des constructions transitives indirectes en français », Travaux de linguistique, $42 / 43$ (111-120).

DUGAS, A. \& CUSSON, L. (1985) "Les prépositions des dénominations complexes", Revue québécoise de linguistique, 4 : 4/1 (159-169).

DUGAS, A. \& MANSEAU, H. (1996) Les verbes logiques, Montréal, Les Éditions Logiques.

FAGARD, B. (2002) «Evolution sémantique des prépositions spatiales de l'ancien au moyen français ", Lingvisticae Investigationes, XXV : 2 (311-338).

- (2006a) Evolution sémantique des prépositions dans les langues romanes: illustrations ou contre-exemples de la primauté du spatial ?, Thèse de l'Université Paris VII.

3 - (2006b) «Les prépositions, du latin aux langues romanes: phénomènes de grammaticalisation ", Modèles linguistiques, 53 (2006: I).

(à par.), «Vers, du français classique au français moderne ou contemporain: l'aboutissement d'une grammaticalisation ? ", in F. Dupuis (éd) Journal de linguistique du Québec, numéro spécial sur la grammaticalisation. 

(47-67).

224 - (1997) «La place du sujet nominal dans les phrases à complément prépositionnel initial », in C. Fuchs (éd) La place du sujet en français contemporain, Louvain-la- 

variation sémantique d'une unité lexicale : le cas du verbe passer ", La locution: entre lexique, syntaxe et pragmatique, coll. StCloud, publication de l'INALF, Paris, Klincksieck (49-68).

- (1999) «La position des groupes prépositionnels dans l'énoncé », in F. Neveu (éd) Phrases : syntaxe, rythme, cohésion du texte, Paris, Sedes (81-96).

FRANCKEL, J.J. (éd) (2003), « Le lexique entre identité et variation », Langue française, $133(28-41)$

FRANCKEL, J.J. (1993) «Il y a lieu de prendre place dans un endroit facilement localisable ", Opérations énonciatives et interprétations de l'énoncé, Paris, Ophrys (209-221).

FRANCKEL, J.J. \& LEBAUD, D. (1991), "Diversité des valeurs et invariance du fonctionnement de en préposition et préverbe ", Langue française, 91 (56-79).

FRANCKEL, J.J. \& PAILLARD, D. (1998), «Les emplois temporels des prépositions : le cas de sur ", Cahiers Chronos, 3 (199-211).

- (1998), « Aspects de la théorie d'Antoine Culioli », Langages, 129 (52-63).

- (1999) "Considérations sur l'antéposition des syntagmes prépositionnels", in C. Guimier (éd) La thématisation dans les langues, Bern, Peter Lang (277-295).

(2006), Prépositions et rection verbale, Paris, Ophrys.

FRANCKEL, J.J., PAILlARD, D. \& SAUNIER, E. (1997), « Modes de régulation de la GAATONE, D. (1976) « Locutions prépositives et groupes prépositionnels. Observations sur la syntaxe de certains groupes prépositionnels », Linguistics, 167 (15-34).

- (2001) "Les prépositions: une classe aux contours flous", Travaux de linguistique, 42/43 (23-31).

7 - (2004) «Les prépositions forment-elles une classe ? », in C. Leclère, E. Laporte, M. Piot \& M. Silberztein (éds) Lexique, Syntaxe et Lexiquegrammaire. Syntax, Lexis \& LexiconGrammar. Papers in honour of Maurice Gross, Amsterdam/Philadelphia, John Benjamins, coll. Lingvisticae Investigationes: supplementa, 24 (211-221).

GAMILLSCHEG, E. (1952) «Präposition und Adverbium im Französischen », Zeitschrift für romanische Philologie, 68 (209-242).

GANDON, F.M. (1994) «Appropriation et syntaxe du français écrit dans la presse de Ouagadougou (Burkina Faso): préposition, rection, pronoms ", Langue française, 104 (70-88).

GARNIER, G. (1993) "Structure du groupe de circonstants », in C. Guimier (éd) 1001 circonstants, Caen, Presses Universitaires de Caen (207-234).

GARRIGUES, M. (1993) «Prépositions et noms de pays et d'îles : une grammaire locale pour l'analyse automatique des textes ", Lingvisticae Investigationes, XVII : 2 (281-305).

- (1995) « Prepositions and the Names of Countries and Islands: a Local Grammar for the Automatic Analysis of Texts », Language Research, 31: 2 (309-334).

GARY-PRIEUR, M.N. (1982) «Adverbe de manière: que signifie cette étiquette?», Lexique, 1 (13-23).

GAATONE, D. (1998), Le passif en français, Paris/Bruxelles, Duculot.

GAWRON, J. M. (1986) "Situations and Prepositions", Linguistics and Philosophy, 9 (327-382). 
265 - (1950) « Animé et inanimé. A propos de qui interrogatif et de qui relatif

266 - (1954) « Tant de royaumes réunis dans une vaste monarchie ", Le français moderne, 22 (96). 
268 - (1961) «Prépositions et conjonctions de subordination en français », Bulletin de la Société Linguistique de Paris, 56 : 1 (86-103).

269 - (1962) Système grammatical de la langue française, Paris, Éditions d'Artrey.

270 - (1970) Études de grammaire et de vocabulaire français, Paris, Éditions A. \& J. Picard. GOVAERT, M. (1972) "L'étoffement de la préposition », Linguistica Antverpiensa, 6 (87-141).

271 GOYENS, M. \& DE MULDER, W. (éds) (2002) Lingvisticae Investigationes, XXV : 2 : Grammaticalisation: le cas des prépositions locatives, Amsterdam/Philadelphia, John Benjamins Publishing Company.

272 GREVISSE, M. (1977) Quelle préposition ?, Paris/Gembloux, Duculot.

273 - (1993), Le Bon Usage, Paris/Louvain-la-Neuve, Duculot (1ère éd. 1936) refondue par A. Goosse.

274 GRIMSHAW, J. (1992), Argument Structure, Cambridge Mass., The MIT Press.

275 GROSS, G. (1981) «Les prépositions composées », in C. Schwarze (éd) Analyse des prépositions, Tübingen, Max Niemeyer Verlag (29-39).

276 - (1984) «Compléments adverbiaux et verbes supports ", Revue québécoise de linguistique, 13 : 2 (123-156).

277 - (1987) Noms composés de type 'Prép N', Rapport de Recherches, nº 1, LLI, Villetaneuse, Université Paris XIII.

278 - (1988a) «Réflexions sur la notion de locution conjonctive », Langue française, 77 (19-36).

279 - (1988b) «Les connecteurs sont-ils des opérateurs? » Opérateurs syntaxiques, cohésion discursive, Actes du $4^{\circ}$ colloque international de linguistique slavo-romane, Copenhague, Nyt Nordisk Forlag Arnold Busck (37-51).

280 - (1991a) Groupes prépositionnels à valeur adjectivale. Rapport de Recherches, $\mathrm{n}^{\circ} 8$, Laboratoire de Linguistique Informatique, Villetaneuse, Université Paris XIII.

281 - (1991b) «Syntaxe du complément de nom », Lingvisticae Investigationes, XV : 2 (255-284).

282 - (1994) "Connecteurs et traitement automatique », TA-TAO: Recherches de pointe et applications immédiates, FMA-Beyrouth et AUPELF-UREF-Québec (287-306).

283 - (1995a) «A quoi sert la notion de partie de discours? », Les classes de mots. Traditions et perspectives, Presses Universitaires de Lyon (217-231).

284 - (1995b) «Enseignement des connecteurs", Les Cahiers de l'Asdifle, 6, Lexique et didactique du français langue étrangère.

285 (1996a) « Semantische Umgebung der Konnektoren », Leuvense Bijdragen, 84 (295-311).

286 - (1996b) «Une typologie sémantique des connecteurs : l'exemple de la cause », Studi italiani di linguistica teorica e applicata, XXV :1, Pise, Pacini Editore (153-179).

287 - (1997) « Du bon usage de la notion de locution », La locution entre langue et usage, ENS Editions Fontenay SaintCloud, coll. Langages (201-223).

288 - (1999a) «Etude lexicale des locutions conjonctives", Mémoires de la Société de Linguistique de Paris, VII (23-40).

289 - (1999b) «Sémantique lexicale et connecteurs », Langages, 136 (76-84). 
290 - (1999c) «Traduire les connecteurs ", L'Atto del Tradurre. Aspetti teorici e Pratici_ella Traduzione, coll. Biblioteca di Cultura, 574, Roma, Bulzoni Editore (7390).

291 - (2000a) «Un complément circonstanciel peut-il devenir sujet ? », Cahiers de l'ILSL, 12 (89-100).

292 - (2000b), « Passifs nominaux et verbes intransitifs », in L. Schosler (éd) Le passif, Actes du colloque international organisé par l'Institut d'Etudes Romanes de l'Université de Copenhague, mars 1998, coll. Etudes romanes, 45 (227-236)

293 - (2001) «Existe-t-il des verbes supports de type Etre Prép? », Par monts et par vaux, Itinéraires linguistiques et grammaticaux. Mélanges de linguistique générale et française offerts au professeur Martin Riegel, Louvain/Paris Editions Peters (197-204).

294 - (2002) « Locutions conjonctives et détermination », Langages, 145 (9-20).

295 - (2003) « Du figement dans les expressions temporelles », Etudes Linguistiques romanoslaves offertes à Stanislaw Karolak, Cracovie, Oficina Wydawnicza, Edukacja (195-202).

296 - (2005) «Les circonstancielles sont des complétives", La syntaxe au cœur de la grammaire, recueil offert en hommage pour le $60^{e}$ anniversaire de Claude Muller, Presses Universitaires de Rennes (121127).

297 - (2006) «Sur le statut des locutions prépositives», Modèles linguistiques, 53 (2006 : I). GROSS, M. (1975), Méthodes en syntaxe, Paris, Hermann.

298 - (1976) «Sur quelques groupes nominaux complexes », in J.C. Chevalier \& M. Gross (éds) Méthodes en grammaire française, Paris, Klincksieck (97-119).

299 - (1986) Grammaire transformationnelle du français - 3. Syntaxe de l'adverbe, Paris, ASSTRIL.

300 - (1990) «La caractérisation des adverbes dans un lexiquegrammaire », Langue française, $86(90-102)$.

301 - (1996) «Les formes être Prép X du français », Lingvisticae Investigationes, XX : 2 (217-270).

302 - (1999) «Sur la définition d'auxilaire du verbe », Langages, 135 (8-21).

303 - (2000) « Verbes à trois compléments essentiels », BULAG - Lexique, Syntaxe, Sémantique. Mélanges offerts à Gaston Gross a l'occasion de son $60^{\text {me }}$ anniversaire (199-211).

304 - (2002) «Les déterminants numéraux, un exemple : les dates horaires », Langages, 145 (21-37).

305 GROUSSIER, M.L. (1980) « Processus de déplacement et métaphore spatiotemporelle », Modèles linguistiques, $2: 1$ (57-106).

306 - (1993) «La double iconicité des prépositions », Faits de langues, 1 (141-150).

307 - (1995) «Opérations et relations de repérage : les prépositions, marqueurs ambivalents mais non ambigus ", in J. Bouscaren, J.J. Franckel \& S. Robert (éds) Langues et langage : Problèmes et raisonnement linguistique, Paris, PUF (899).

308 - (1997) « Notions de procès, prédication et prépositions ", in C. Rivière \& M.L. Groussier (éds) La Notion, Paris, Ophrys (137-145).

309 - (1997) «Prépositions et primarité du spatial: de l'expression de relations dans l'espace à l'expression de relations non-spatiales », Faits de langues, 9 (221-234).

310 - (2003) «Les prépositions comme parties de prédicats ou prédicats annexes », in J. Pauchard (éd) Les Prépositions dans la rection des verbes (domaine anglais), Reims, PU de Reims (61-83). 
311

312

GRUBER, J. (1965), Studies in Lexical Relations, PhD thesis, Massachusetts Institute of Technology.

GUENTCHEVA, Z. (2001) "Sémantique préfixale et fonctions préfixales", Etudes cognitives, 4, Varsovie, Instytut Slawistyki, Polska Akademia nauk (49-64).

- (2005) «A propos de la compositionnalité et du fonctionnement de la préverbation dans le domaine slave ", in P. Dendale (éd) Le mouvement dans la langue et la métalangue, Université de Metz, coll. Recherches linguistiques, 27 (217-242).

GUILLAUME, G. (1975) Le problème de l'article et sa solution dans la langue française, Paris, Nizet (1 ${ }^{\text {ère }}$ éd. 1919).

GUILLEMARD, C. (2003) 'L'affaire est dans le sac' et nouveaux mystères des expressions françaises, Paris, Bartillat.

GUILLET, A. (1984) «Prépositions de lieu et verbes supports ", Revue Québécoise de Linguistique, $13: 2$ (59-94).

GUILLET, A. \& LECLÈRE, C. (1992), La structure des phrases simples en français. Constructions transitives locatives, Genève/Paris, Droz.

GUIMIER, C. (1981) Prepositions : An Analytical Bibliography, Amsterdam, John Benjamins.

- (1993a) 1001 circonstants, Caen, Presses Universitaires de Caen.

- (1993b) «L'établissement d'un corpus de circonstants », in C. Guimier (éd) 1001 circonstants, Caen, Presses Universitaires de Caen (11-45).

- (1993c) «Les circonstants en phrase attributive », in C. Guimier (éd) 1001 circonstants, Caen, Presses Universitaires de Caen (127-157).

2 - (2005), «Les adverbes en -wise de l'anglais moderne : rôles sémantiques », in J. Goes (éd) L'adverbe : un pervers polymorphe, Arras, Artois Presses Université (107-126).

GUNNARSON, K.A. (1972) Le complément de lieu dans le syntagme nominal (Etudes romanes de Lund, 20), Lund, Gleerup.

- (1982) "Trois constructions à dépendance entre sujet et $p p »$, Lingvisticae Investigationes, $\mathrm{VI}: 1$ (45-81).

- (1986) «Loin de X, près de X et parallèlement à X : syntagmes prépositionnels, adjectivaux ou adverbiaux ", Le français moderne, $54: 1 / 2$ (123).

6 HAGEGE, C. (1975) Le problème linguistique des prépositions et la solution chinoise (avec un essai de typologie à travers plusieurs groupes de langue), Louvain, Peeters.

7 - (1997) «Les relateurs comme catégorie accessoire et la grammaire comme composante nécessaire », Faits de langues, 9 (19-27).

HAILLET, P.P. (2002), Le conditionnel en français: une approche polyphonique, Paris, Ophrys, coll. L'essentiel.

HAILLET, P.P. (éd) (2004), Langue française $\mathrm{n}^{\circ} 142$ : Procédés de modalisation : l'atténuation, Paris, Larousse.

HAMMA, B. (2004), « Par exemple : l'expression de l'altérité dans l'acte d'exemplification ", Revue de Sémantique et Pragmatique, 15/16 (155-181).

- (2005), L'invariant sémantique de la préposition 'par' à travers les distributions syntaxiques et lexicales, Thèse de doctorat de l'Université Paris X-Nanterre, sous la direction de Danielle Leeman. 
332 - (2006a), "L'expression de la cause à travers le prisme de la préposition par », in S. Hamon \& M. Amy Hamon (éds) Linx, 53 : La notion de cause : approche pluridisciplinaire.

333 - (2006b), « La préposition par, génératrice de polylexicalités », in A. BalibarMrabti \& C. Vaguer (éds) Linx, 54 : Le semifigement.

334 - (1970), Papers in structural and transformational linguistics, vol. 1, Holland, D. Reidel Publishing Company.

335 HARRIS, Z. (1947), (Methods in) Structural Linguistics, The University of Chicago Press, Phoenix Books.

336 HATHOUT, N. (1989) « Prépositions de localisation externe dans le plan », Rapport L.S.I., Toulouse.

337 HERSKOVITS, A. (1986), Language and spatial cognition: an interdisciplinary study, Cambridge, Cambridge University Press.

338 HERSLUND, M. (1988a), Le datif en français, Louvain/Paris, Peeters.

339 - (1988b), « On Valence and Grammatical Relations » in F. Sorensen (éd) Valency, coll. Copenhagen Studies in Language, 11 (3-34).

340 - (1990), «Les verbes inaccusatifs comme problème lexicographique ", Cahiers de lexicologie, 56/57 (35-44).

341 - (1994), "Valence et relations grammaticales », Mélanges Lucien Tesnière, Linguistica, XXXIV : 1 (109-117).

342 - (1997), « Les relations syntaxiques dans une approche valentielle », in R. Lorenzo (éd) Actas do XIX Congreso Internacional de Lingüística e Filoloxía Románicas, Universidade de Santiago de Compostela, 1989. Sección I. Lingüística Teórica e Lingüística Sincrónica, Coruña, Fundación « Pedro Barrié de la Maza, Conde de Fenosa » (555-562).

343 - (2001), «L'actant fondamental et les verbes symétriques et réfléchis de l'ancien français ", in L. Schosler (éd) La valence, perspectives romanes et diachroniques, Stuttgart, Franz Steiner Verlag, coll. ZFSL Beihefte, 30 (34-42).

344 HERSLUND, M. \& BARON, I. (1999), "Dimensions spatiales du verbe avoir ", in J.R. Klein, B. Lamiroy \& J.M. Pierret (éds) Théorie linguistique et applications informatiques. Actes du $16^{e}$ Colloque européen sur la grammaire et le lexique comparé, coll. Cahiers de l'Institut de Linguistique de Louvain, 24 (49-61).

345 - (2001), «Introduction. Dimensions of Possession », in I. Baron, M. Herslund \& F. Sorensen (éds) Dimensions of Possession, Amsterdam/Philadelphia, Benjamins (1-25).

346 HERSLUND, M. \& SORENSEN, F..(1994), «A Valence Based Theory of Grammatical relations ", in E. EngbergPedersen et al. (éds) Function and Expression in Functional Grammar, Berlin/New York, Mouton de Gruyter (81-95).

347 - (1996), «Introduction. Discussion », in N. DavidsenNielsen (éd) Sentence Analysis, Valency, and the Concept of Adject, coll. Copenhagen Studies in Language, 19 (913 \& 143-157). HENRY, A. (1966) «Il y a préposition?», in F. Desonay (éd) Mélanges de grammaire française offerts à M. Maurice Grevisse pour le trentième anniversaire du 'Bon Usage', Gembloux, Duculot (207-213).

349 HICKMANN, M., HENDRIKS, H. \& ROLAND, F. (1998) «Référence spatiale dans les récits d'enfants français : perspective interlangues », Langue française, 118 (104-123). 
HILL, C. A. (1991) « Recherches linguistiques en orientation spatiale », Communications, 53 (171-207).

HOMMA, Y. (2003), « A propos de quelques emplois « marginaux » de PAR», Colloque Le langage, ce singulier, ce particulier, Université de Tsukuba, actes à paraître sous la direction de S. Aoki, Japon.

IBRAHIM, A. H. (1999) «Les prépositions comme traces ou équivalents d'un support ", Revue de Sémantique et Pragmatique, 6 (89-102).

ILINSKI, K. (2000) La syntaxe atypique de la préposition française, Thèse de doctorat, Université Paris IV.

- (2001) «De la préposition. Incidence et incorporation», Actes du $8 »^{n e}$ colloque international de psychomécanique du langage (Seyssel), Paris, Honoré Champion (295-302).

- (2003) La préposition et son régime. Etude des cas atypiques, Paris, Honoré Champion.

JACKENDOFF, R. (1972), Semantic interpretation in Generative Grammar, chap. VI, MIT Press.

JACKENDOFF, R. \& LANDAU, B. (1992), "Spatial language and spatial cognition », in R. Jackendoff (éd) Languages of the mind, Cambridge Mass., The MIT Press (99-124)

JALENQUES, P. (2002), «Etude sémantique du préfixe RE en français contemporain : à propos de plusieurs débats actuels en morphologie dérivationnelle ", Langue française, 133 (74-90).

JONASSON, K. (1986), "L'article indéfini générique et la structure de l'énoncé ", Travaux de linguistique et de littérature, XXIV : 1 (309-345).

IORDANSKAJA, L. \& ARBATCHEWSKY-JUMARIE, N. (2000) "Quatre prépositions causales du français. Leur sémantisme et cooccurrence ", Lingvisticae Investigationes, XXIII : 1 (115-159).

JAEGGI, A. (1956) Le rôle de la préposition et de la locution prépositive dans les rapports abstraits en français moderne, Berne, Francke.

JEANES, R. W. (1972) «Problems of French grammar and usage: $n^{\circ} 15 »$ (Preposition and article), The Canadian Modern Language Review, 18: 3 (48-55).

JENKINS, F. M. (1980) « Mixing Adjectives and Prepositional Phrases in Postnominal Position ", The French Review, 53 (696-702).

KAMINKER, J.P. (1979) «(SP) », Langue française, 41 (111-128).

KAMPERS-MANHE, B. (2001) «Le statut de la préposition dans les mots composés ", Travaux de linguistique, 42/43 (97-109).

KEMMER, S. \& BATZEEV SHYLDKROT, H. (1996) "The semantics of 'empty prepositions' in French", in E. Casad (éd) Cognitive Linguistics in the Redwoods. The Expansion of a News Paradigm in Linguistics, Berlin/New York, Mouton de Gruyter (347-388).

KHAMMARI, I. (2004a), "En préfixe et en préposition: une seule forme, un seul sens? », Linx, 50 (169-178).

- (2004b), «Etude de la construction X être en Vn E», in C. Vaguer \& B. Lavieu (éds) Le verbe dans tous ses états. Grammaire, sémantique, didactique, Namur \& CEDOCEF, Presses Universitaires de Namur, coll. Diptyque, 2 (135-148). 
KING, R. \& ROBERGE, Y. (1990) «Preposition Stranding in Prince Edward Island French », Probus, 2: 3 (351-369).

KLEIBER, G. (1990), La sémantique du prototype. Catégories et sens lexical, Paris, PUF.

- (1993), «Prototype et prototypes: Encore une affaire de famille» in D. Dubois (éd) Sémantique et cognition. Catégories, prototypes, typicalités, Paris, CNRS (103-129).

372 - (1994), « Métaphore : le problème de la déviance », Langue française, 101 (35-56).

373 KLEIN, W. \& NÜSE, R. (1997) «La complexité du simple : l'expression de la spatialité dans le langage humain », in M. Denis (éd) Langage et cognition spatiale, Paris, Masson (1-23).

KOPECKA, A. (2004), Etude typologique de l'expression de l'espace : localisation et déplacement en français et en polonais, Thèse de doctorat, Université Lumière Lyon 2.

KOTSCHI, T. (1981) "Verbvalenz im Französischen », in T. Kotschi (éd) Beiträge zur Linguistik des Französischen, Tübingen, Narr (80-122).

KUPFERMAN, L. (1981) «Le mouvement des groupes prépositionnels ", Lingvisticae Investigationes, $\mathrm{V}: 1$ (39-73).

377 - (1991), «Structure événementielle de l'alternance un / devant les noms humains attributs », Langages, 102 (52-75).

378 - (2001) «La préposition française dans tous ses états », Travaux de linguistique, 42/43 (78).

379 - (éd) (2002) Scolia ${ }^{\circ} 15$ : La préposition dans tous ses états, 4, Strasbourg.

KUPFERMAN, L., KATZ, E. \& ASNES, M. (éds) (2001) Travaux de linguistique $\mathrm{n}^{\circ} 42 / 43$ : La préposition, Bruxelles, De Boeck \& Larcier.

381 - (2002) Travaux de linguistique $\mathrm{n}^{\circ} 44:$ La préposition (suite), Bruxelles, De Boeck \& Larcier.

KWON-PAK, S.N. (1997a) «Les prépositions, révélateurs de polysémie nominale », Sémiotiques, 13 (31-40).

383 - (1997), Les prépositions spatiales : sur quelques emplois de 'par', Thèse de l'Université de Strasbourg, sous la direction de G. Kleiber.

384 L'HOMME, M.C. (1996) «Sélection des prépositions dans leion das termes complexes Nom (Prép) Nom à partir de leur structure conceptuelle ", Cahiers de lexicologie, 68 (25-43).

LABELLE, M. (1992) «La structure argumentale des verbes locatifs à base nominale ", Lingvisticae Investigationes, XVI : 2 (267315).

386 LAFONT, R. (1990) «Les particules directionnelles, ou l'aventure topologique de la langue ", in R. Lafont (éd) Le dire et le faire, Montpellier III, coll. Langue et Praxis (227-249).

387 LAMIROY, B. (1999) «Auxiliaires, langues romanes et grammaticalisation », Langages, $135(33-45)$.

LANG, E. (1991a) Die französischen Präpositionen. Funktion und Bedeutung, Heidelberg, Carl Winter Universitätsverlag.

389 - (1991b) « A twolevel approach to projective prepositions », in G. Rauh (éd) Approaches to Prepositions, Tübingen, Günter Narr Verlag (127-168).

390 LANGACKER, R. W. (1987), Foundations of cognitive grammar, vol.1: theoretical prerequisites, Stanford, Stanford University Press. 
391 - (1991a), Concept, image and symbol: the cognitive basis of grammar, Berlin/New York, Mouton de Gruyter.

392 - (1991b), Foundations of cognitive grammar, vol.2: descriptive application, Stanford, Stanford University Press.

393 LANGLAIS, A. (1996) Prépositions et particules adverbiales: Versatilité sémantique et fonctionnalité grammaticale - Etude de linguistique contrastive, Université Paris IIISorbonne Nouvelle.

394 LARSEN, E. S. (1987a) Langages n 86 : Actualité de Brendal, Paris, Larousse.

395 - (1987b) « Les maillons du langage. La sémantique rationnelle de Brandal : Théorie des prépositions », Langages, 86 (95-110).

396 LAUR, D. (1989) «Sémantique du déplacement à travers une étude de verbes et de prépositions du français ", Cahiers de grammaire, 14 (65-84).

397 - (1991) Sémantique du déplacement et de la localisation en français : une étude des verbes, des prépositions et de leurs relations dans la phrase simple, Thèse de doctorat, Université de Toulouse Le Mirail.

398 - (1993) «La relation entre le verbe et la préposition dans la sémantique du déplacement ", Langages, 110 (47-67).

399 LAUR, D. \& SARDA, L. (1999) «Le rôle des prépositions dans l'expression du déplacement: comparaison des constructions transitives directes et indirectes ", Recherches en linguistique et en psychologie cognitive, 11, Presses Universitaires de Reims (131-151).

400 LAURENDEAU, P. (1987) «Pour une étude non contrastive des marqueurs linguistiques dans les vernaculaires du français », Bulletin de linguistique appliquée et générale, 13 (51-103).

401 LAUWERS, P. (2001) «Le complément pris entre deux syntaxes ", in E. Aïm et al. (éds) Actes des sixièmes rencontres des doctorants en linguistique de Paris (83-88).

402 - (2002) «Forces centripète et centrifuge. Autour du complément circonstanciel dans la grammaire 'traditionnelle' de la première moitié du $\mathrm{XX}^{\text {ème }}$ siècle ", Travaux de linguistique, 44 (115-142).

403 LAVIEU, B. (2006), « De la difficulté de distinguer entre groupes prépositionnels régis et non régis ", Modèles linguistiques, 53 (2006: I).

404 LAY, M.H. Une lecture du phénomène de la composition lexicale : description des structures et comportements des composés syntaxiques commençant par une préposition, Thèse de l'Université Paris V.

405 LE DRAOULEC, L. \& PERY-WOODLEY, P.P. (2005) « Encadrement temporel et relations de discours ", Langue française, 148 (45-60).

406 LE PESANT, D. (2006) «Esquisse d'une classification syntaxique des prépositions simples du français ", Modèles linguistiques, 53 (2006: I).

407 LE QUERLER, N. (1993) « Les circonstants et la position initiale », in C. Guimier (éd) 1001 circonstants, Caen, Presses Universitaires de Caen (159-184).

408 - (2005) «Les périphrases verbales d'immixtion. Schéma actanciel, complémentation et organisation thématique », in H. BatZeev Shyldkrot \& N. Le Querler (éds) Les périphrases 
verbales, Amsterdam/Philadelphia, John Benjamins, coll. Lingvistiace Investigationes: Supplementa, 25 (229-243).

LEEMAN, D. (éd) (1979) Langue française $n^{\circ} 41$ : Sur la grammaire traditionnelle, Paris, Larousse.

410 - (1990) Langue française $n^{\circ} 86$ : Sur les compléments circonstanciels, Paris, Larousse.

411 - (1998) Les circonstants en question(s), Paris, Kimé.

412 LEEMAN, D. (1982) «La difficulté d'élaborer une leçon de grammaire quand on a été initié à la linguistique », Langue française, 55 (45-65).

413 - (1985/1986) «A propos du complément circonstanciel », Travaux de linguistique, 12/13 (177-193).

414 - (1987), « A ma grande surprise... », Revue québécoise de linguistique, $1: 2$ (415-434).

415 - (1994) Les fautes de français existent-elles ?, Paris, Le Seuil.

416 - (1994), «Si j'aurais su, j'aurais pas venu. Remarques sur les auxiliaires, la transitivité et l'intransitivité », Le Gré des Langues, 7 (48-63).

417 - (1995) Compléments circonstanciels et prépositions, Dossier présenté pour l'habilitation à diriger des recherches, Tomes II/III, Université de Paris X-Nanterre.

418 - (1995), «Pourquoi peut-on dire Max est en colère mais non *Max est en peur? Hypothèses sur la construction être en $N »$, Langue française, 105 (55-69).

419 - (1996a), « Le « sens » et l'« information » chez Harris », Du dire et du discours. Hommages à Denise Maldidier, Linx (numéro spécial), (209-220).

420 - (1996b), « Vêture, coiffure, chaussures et autres... coquetteries. La construction être en et les noms de vêtement ", Sémiotique, phénoménologie, discours. Du corps présent au sujet énonçant. Hommages à JeanClaude Coquet, Paris, L'Harmattan (79-89)

421 - (1997a) "Définir une préposition : hypothèses et perplexités ", Revue de Sémantique et Pragmatique, 2 (183-199).

422 (1997b), Sur la préposition en », Faits de langues, 9 (135-143).

423 - (1998a) «La préposition comme catégorie prédicative », in M. Forsgren, K. Jonasson \& H. Kronning (éds) Prédication, Assertion, Information, Acta Universitatis Upsaliensis, Uppsala, coll. Studia Romanica Upsaliensia, 56 (327-334).

424 - (1998b) «La métaphore dans la description des prépositions", Verbum, XX : 4 (435-458).

425 - (1998)c, Les circonstants en question(s), Paris, Kimé.

426 - (1999a) « La préposition : un « auxiliaire » du nom ? », Langages, 135 (75-86).

427 - (1999b) «L'unité lexicale dans la perspective harrissienne », Linx, 40 (117-136).

428 - (1999)c, «Dans un juron, il sauta sur ses pistolets. Aspects de la polysémie de dans », Revue de Sémantique et Pragmatique, 6 (71-88).

429 - (2000a) «Compléments circonstanciels ou appositions ? ", Langue française, 125 (18-29).

430 - (2000b) «Compte rendu de l'ouvrage de J. Cervoni (1991) », L’information grammaticale, 87 (66-67).

431 - (2001)a «Quand les formes informent: de la grammaire à la sémantique », Le français aujourd'hui, 135 (12-19).

432 - (2001)b, «Tout contre vs Très contre », Travaux de Linguistique, 42/43 (241-252) 
433 - (2002) «La construction du sens par la grammaire », Cahiers de l'Institut de linguistique et des sciences du langage, 13 (17-26).

434 - (2003a) «Complémentation verbale et préposition», Colloque Modèles Syntaxiques, Bruxelles, U.L.B., à paraître.

435 - (2003b), "Les adjectifs construits avec contre», in P. Péroz (éd) Contre 'Identité sémantique et variation catégorielle', Université de Metz, coll. Recherches linguistiques, 26 (113-133).

436 - (2003c), « Nom et verbe au regard de la préposition contre », in C. Brion \& E. Castagne (éds) Nom et verbe: catégorisation et référence, Reims, Presses Universitaires de Reims, coll. Recherches en Linguistique et Psychologie cognitive, 18 (139-159).

437 - (2005a), «La préposition jusque », in P. Dendale (éd) Le mouvement dans la langue et la métalangue, Université de Metz, coll. Recherches linguistiques, 27 (103-119).

438 - (2005b), « Un nouvel auxiliaire : aller jusqu'à », in H. BatZeev Shyldkrot \& N. Le Querler (éds) Les périphrases verbales, Amsterdam/Philadelphia, John Benjamins, coll. Lingvistiace Investigationes: Supplementa, 25 (361-377).

439 - (2005c), Jusque. Conférence d'Osaka (décembre 2005).

440 - (2006a) "La préposition française: caractérisation syntaxique de la catégorie", Modèles linguistiques, $53(2006,1)$.

441 LEEMAN, D. \& VAGUER, C. (éds) (2006a) Modèles linguistiques n 53 : La préposition en français.

442 LEGENDRE, G. \& SORACE, A. (2003), « Auxiliaires et intransitivité en français et dans les langues romanes ", in D. Godard (éd) Les langues romanes: problèmes de la phrase simple, Paris, Editions du C.N.R.S. (185-234).

443 LE GUERN, M. (1973), Sémantique de la métaphore et de la métonymie, Paris, Larousse.

444 LEFEUVRE, F. \& NICOLAS, D. (2003) « La phrase nominale existentielle et la distinction aspectuelle télique / atélique ", Revue de Sémantique et Pragmatique, 14 (157-172).

445 LEMARECHAL, A. (1991) «Transitivité et théories linguistiques : modèles transitivistes contre modèles intransitivistes ", Linx, 24 (67-94).

446 - (1995) « Actants ou arguments? », in F. MadrayLesigne \& J. RichardZapella (éds) Lucien Tesnière aujourd'hui, Louvain, Peeters (165-174).

447 - (1997) «Séries verbales et prépositions : incorporation et décumul des relations », Faits de langues, 9 (109-118).

448 LETOUBLON, F. (1990) « Sur les genoux des dieux », Langue française, 86 (22-29).

449 LEVIN B. \& RAPPAPORT HOVAV, M. (1995), Unaccusativity. At the Syntax - Lexical Semantics Interface, Cambridge Mass., The MIT Press

450 LONG, M. E. (1974) «Preposition and postpositions: some remarks on French Infinitives", in J. Campbell et al. (éds) Linguistic Studies in Romance Linguistics, Washington, D. C., Georgetown University Press (237-253).

451 LOWE, R. (1997) « Le caractère diastématique du régime d'incidence de la préposition ", in P. De Carvalho \& O. Soutet (éds) Psychomécanique du langage. Problèmes et perspectives, Paris, Honoré Champion (213-222).

452 MAEDER, C. (1998) «Caractéristiques communes à l'expression des notions spatiales et temporelles en langue des signes française », Verbum, XX : 2 (177-188). 
MAILLOT, C. (2000) « Traduction automatique de la configuration [verbe + préposition spatiale + nom de pays] », BULAG, 25 (145-154).

MANDIC, R. \& PATOZ, E. (1995/1996) « Reconnaissance automatique de l'adverbe, de la préposition, de la locution adverbiale et de la locution prépositive dans un énoncé ", BULAG, 21 (195-204).

MARANTZ, A. P. (1984), On the Nature of Grammatical relations, Cambridge Mass., The MIT Press.

MARCHELLONIZIA, C. (2002) «Prépositions françaises en diachronie : une catégorie en question », Lingvisticae Investigationes, XXV : 2 (205-221).

MARQUE-PUCHEU, C. (1995a) "Variété des marques syntaxiques et lexicales de l'aspect : au fil de N », Lingvisticae Investigationes, XIX : 2 (369-386).

- (1995b) Structures prépositionnelles semifigées 'Prép Ci de N2': verbes supports associés et structure interne, Thèse de doctorat, Université Paris VIII.

- (1996) «Les alternances prépositionnelles : des substitutions très contraintes », Linx, $34 / 35$ (219-231).

- (2001a) «Les locutions prépositives : du spatial au non-spatial », Langue française, 129 (35-53).

- (2001b) «La sélection des noms dans la locution au bord de $N$ et le concept analytique de 'crise' ", Le français moderne, $69: 2$ (183-198).

MARTINOT, C. (1998) «Développement de la construction argumentale de trois verbes essentiels : mettre, prendre, donner ", Langue française, 118 (61-83).

- (2000) «Etude comparative des processus de reformulation chez des enfants de 5 à 11 ans ", Langages, 140 (92-123).

MATSUMOTO, Y. (2003), « Typologies of lexicalization patterns and event integration: clarifications and reformulations", in Chiba, Shuji et al. (éds) Empirical and theoretical investigations into language: a festschrift for Masaru Kajita, Tokyo, Kaitakusha (403-418).

MAUREL, D. (1990) «Adverbes de date: étude préliminaire à leur traitement automatique, Lingvisticae Investigationes, XIV : 1 (31-63).

- (1993) «Reconnaissance automatique d'un groupe nominal prépositionnel. Exemple des adverbes de date », Lexique, 11 (147-161).

MAUREL, J.P. (1990) « Examen circumstantiarum », Langue française, 86 (30-36).

McA'NULTY, J. (1977) «La dérivation de quelques prépositions du français », Cahiers de linguistique, 2 (111-131).

MEILLET, A. (1912) "L'évolution des formes grammaticales", Scientia (Rivista di Scienza), 12, 26:6, in (réed. 1982) Linguistique historique et linguistique générale, Genève/ Paris, Slatkine/Champion (130-148).

MELIS, L. (1977) Esquisse d'une classification des compléments circonstanciels, Thèse pour le Doctorat, Antwerpen.

- (1983) Les circonstants et la phrase. Etude sur la classification et la systématique des compléments circonstanciels en français moderne, Louvain, Presses Universitaire de Louvain.

- (1985) «Un type particulier de complément circonstanciel: le complément de phrase », Enjeux, 7 (72-85). 
473 - (1996) « The dative in Modern French », in W. Van Belle \& W. Van Langendonck (éds) The Dative 1. Descriptive Studies, Amsterdam, Benjamins (39-72).

474 - (1998) «Réflexions sur la structure syntaxique du syntagme nominal », in A. Englebert et al. (éds) La ligne claire, de la linguistique a la grammaire, Louvain-La-Neuve/Paris, Duculot (99-116).

475 - (2000) «La préposition en interposition », in A. Englebert et al. (éds) Actes du XXII ème Congrès International de Linguistique et de Philologie Romanes, VI, Tübingen, Niemeyer (353-359).

476 - (2001) «La préposition est-elle toujours la tête d'un groupe prépositionnel ? », Travaux de linguistique, 42/43 (11-22).

477 - (2003a) La préposition en français, Paris, Ophrys, coll. L'essentiel français.

478 - (2003b) « Les quantificateurs approximatifs de type prépositionnel », Verbum, XXV : 1 (5-24).

479 - (2003c) «Le groupe prépositif comme déterminant du nom », in P. Haderman et al. (éds) La syntaxe raisonnée-Mélanges de linguistique générale et française offerts à Annie Boone, Bruxelles/Paris, De Boeck/Duculot (235-250).

480 - (2004a) «La suite 'préposition adjectif' et la définition syntaxique de la préposition », Leuven, KUL, Departement Linguistiek.

481 - (2004b), La préposition en français, Paris, Ophrys.

482 MELIS, L. \& SWIGGERS, P. (1993) «Chronique de linguistique générale et française (V) : les prépositions », Travaux de linguistique, 26 (165-188).

483 MENDES, A. (1999) «Analyse aspectuelle et structure lexico-conceptuelle des verbes psychologiques. Transitivité et langues romanes, de l'objet direct à l'objet indirect », Verbum, XXI : 1 (25-36).

484 MEUNIER, A. (1999) « Auxiliaire de modalité et complément adverbial », Langages, 135 (108-122).

485 MICULESCU, A. (1959) «Sur l'objet direct prépositionnel dans les langues romanes », Recueil d'études romanes, Bucarest, Ed. Acad. Rep. Pop. Roum. (167-185).

486 MILNER, J.C. (1977) « A propos des génitifs adnominaux en français », in C. Rohrer (éd) Actes du Colloque Franco-allemand de Linguistique Théorique, Tübingen, Niemeyer (61-108).

487 - (1989), Introduction a une science du langage, Paris, Seuil.

MOESCHLER, J. \& REBOUL, A. (1994), Dictionnaire encyclopédique de pragmatique, Paris, Seuil.

489 MOIGNET, G. (1973) «Incidence verbale et transitivité », Travaux de linguistique et de littérature, 11 (363-379).

490 - (1974a) «Sur la 'transitivité indirecte' en français », Travaux de linguistique et de littérature, $12: 1$ (281-299).

491 - (1974b) Étude de psycho-systématique française, Paris, Klincksieck

492 - (1975) « Incidence et attribut du complément d'objet », Travaux de linguistique et de littérature, 13 (253-270).

493 - (1981) Systématique de la langue française, Paris, Klincksieck. 

la préposition sur », in D. Paillard \& O. Seliverstova (éds) Issledovanija po semantike predlogov (Recherches sur la sémantique des prépositions), Moscou, Russkie Slovari (152-189).

506 - (2002) « Prépositions et rection verbale », Travaux de linguistique, 44 (51-67).

507 - (2003), «A propos de l'ambivalence catégorielle préfixe / préposition: le cas de contre ", in P. Péroz (éd) Contre, identité sémantique et variation catégorielle, Université de Metz, coll. Recherches linguistiques, 26 (153-172).

MOLINIER, C. (1990) «Les quatre saisons. A propos d'une classe d'adverbes temporels », Langue française, 86 (46-50).

MORGENSTERN, A. \& SEKALI, M. (1997) «L'acquisition des premières prépositions chez un enfant francophone », Faits de langues, 9 (201-210).

MORINIERE, C. (de la) (1880) French prepositions and idioms, Paris, Galignani.

(éd) Analyse des prépositions, Tübingen, Max Niemeyer Verlag (88-110).

NEVEU, F. (1998), Etudes sur l'apposition, Paris, Honoré Champion.

NOAILLY-LE BIHAN, M. (1982) "Côté, question et quelques autres", Lingvisticae Investigationes, VI : 2 (333-343).

NOAILLY, M. (2006) «Quoi de neuf côté prépositions?», Modèles linguistiques, 53 (2006:I).

(1982), «Côté, Question, et quelques autres », Lingvisticae Investigationes, VI : 2 (333-343). moderne ", in P. Nykrog \& H. Sörensen (éds) Etudes romanes dédiées a Andreas Blinkenberg a l'occasion de son $60^{\text {ème }}$ anniversaire, Copenhague, Munksgaard (127-143). (277-316). Wratislaviensia, 42 (17-32)

PAPAHAGI, C. (2005), Les prépositions de la trajectoire en français et en roumain. Etude synchronique et diachronique, Thèse de doctorat, Université Paris III - Sorbonne Nouvelle.

PELLEN, R. (1976) «Le poème du Cid étudié à l'ordinateur. Le système prépositionnel », Revue de linguistique romane, 40 (8-34).

PÉROZ, P. (2002), « La préposition contre à partir de la valeur " d'échange ", variation sémantique et régularités des opérations linguistiques », Scolia, 15 (107-122).

- (2003), «A propos de la valeur d'opposition de contre», dans Recherches linguistiques, 26, 2003, "CONTRE Identité sémantique et variation catégorielle », P. Péroz éd., Université de Metz (135-152).

PERRET, M. (1978) «L'espace d'une 'vie' et celui d'une langue. Combinatoire des expressions locatives dans la 'Vie de saint Louis' de Joinville et dans la langue du XI ${ }^{\text {ème }}$ au XVI ${ }^{\text {ème }}$ siècle », Langue française, 40 (18-31). 
513 Keele University, England. Nottingham Linguistic Circular, $5: 2$ (26-30). - apposition attributive », in L. Tasmowski \& A. ZribiHertz (éds) De la musique à la linguistique. Hommages à Nicolas Ruwet, Ghent, Blandijnberg 2, coll. Communication \& Cognition (426-439).

PIERART, B. \& COSTERMANS, J. (1979) « A multidimensional analysis of Some French Prepositions of Space Localization », International Journal of Psycholinguistics, 6 (4557).

517 PINCHON, J. (1966) «Prépositions et locutions prépositives », Le Français dans le monde, 44 (51-52).

518 - (1971) Les pronoms adverbiaux EN et Y, Paris, Hachette.

519 - (1980) «Syntagme prépositionnel et adjectif de relation », Cahiers de lexicologie, 37 (91-100). (141160).

521 - (2001) « Relations entre prépositions et conjonctions ? L'apport de la comparaison en langues romanes », Travaux de linguistique, 42/43 (71-81).

522 - (2005) «Sur la nature des fausses prépositions sauf et excepté », French Language Studies, 15 (297-314).

523 PLANTIN, C. (1990) « La cause du brevet », Langue française, 86 (11-21).

524 POIBEAU, T. \& MAUREL, D. (1995) « A la fin de préposition ou déterminant complexe dans les adverbiaux de temps ? », Cahiers de grammaire, 20 (101-111). POP, L. (2005) « Thématiser en subordination... ou de quelques structures prépositives et conjonctives de thématisation", in M. Tenchea \& A. Tihu (éds) Prépositions et conjonctions de subordination. Syntaxe et sémantique, Timisoara, Editura Excelsior Art (189-206). (77-93).

527 - (2001a) «Prépositions composées et dictionnaires bilingues. Le traitement des propriétés discursives ", Cahiers de lexicologie, 79 (141-163).

528 - (2001b) «Au sujet de et A propos de - une analyse lexicographique, discursive et linguistique », Travaux de linguistique, $42 / 43$ (171-181).

529 - (2005a) "Les marqueurs de thématisation: des thèmes phrastiques et textuels", Travaux de linguistique, 51 (55-84).

530 - (2005b), « Les séquences thématiques », Langue française, 148 (111-126).

531 POTTIER, B. (1950) "Prépositions et conjonctions en français ", Documents pédagogiques, Supplément au n ${ }^{\circ} 21$ de l'Education Nationale, Paris.

532 - (1955 in 1962), Systématique des éléments de relation. Étude de morphosyntaxe structurale romane, Paris, Klincksieck. 
533 - (1957) « Sur la nature du cas et de la préposition : questions de méthodes », Mélanges de linguistique et de littérature romances offerts à la mémoire d'Istvan Franck, Universität des Saarlandes (546-551).

534 - (1960) «L'objet direct prépositionnel: faits et théories », Studii si Cercetäri Lingvisitice, 11 (673-676).

535 - (1961) «Sur le système des prépositions », Le français moderne, 29 : 1 (1-6).

536 - (1962) Systématique des éléments de relation. Etude de morphosynta Moeschler, j. \& Reboul, a. (1994), Dictionnaire encyclopédique de pragmatique, Paris, Seuil. xe structurale romane, Paris, Klincksieck.

537 - (1974) Linguistique générale, théorie et description, Paris, Klincksieck.

538 - (1992a) Sémantique générale, Paris, Presses Universitaires de France.

539 - (1992b) Théorie et Analyse en Linguistique, Paris, Hachette Supérieur (1 ère éd. 1987).

540 - (1997) «Le cognitif et la linguistique dans l'expression des relations », Faits de langues, 9 (29-38).

541 - (2001) Représentations mentales et Catégorisations linguistiques, Louvain/Paris, Éditions Peeters, coll. Bibliothèque de l'information grammaticale, 47.

542 PREVOST, S. (2003) «Les compléments spatiaux : du topique au focus en passant par les cadres ", Travaux de linguistique, 47 (51-77).

543 RAASCH, A. (1968) La préposition dans la phrase: un guide pratique, Frankfurt am Main, Diesterweg.

544 RAUH, G. (1991) Approaches to Prepositions, Tübingen, Günter Narr Verlag.

545 (1994) « Prépositions et rôles : points de vue syntaxique et sémantique », Langages, 113 (45-78).

546 REGARD, P. F. (1918) Contribution à l'étude des prépositions dans la langue du Nouveau Testament, Thèse, Paris.

547 REMI-GIRAUD, S. \& ROMAN, A. (1998) Autour du circonstant, Lyon, Presses Universitaires de Lyon.

RENAUD, F. (2006) Temps, durativité, télicité, Paris/Louvain, Editions Peeters.

RIEGEL M., PELLAT J.C. \& RIOUL R. (1994), Grammaire méthodique du français, Paris, Presses Universitaires de France, coll. Linguistique nouvelle

REYELT, R. (1911), Uber den Gebrauch und die begriffliche Entwicklung der französischen Präposition 'vers', 'envers', 'devers', 'par devers', 'contre', 'encontre' und 'ä l'encontre de', Diss. , University of Göttingen.

RIIHO, T. (1980) « De la formation des systèmes prépositifs romans », Neuphilologische Mitteilungen, 81 (353-360).

552 RITZKE, J. (1979) «Präpositionalgruppen in der automatischen Syntaxanalyse des Französischen", in M. Van de Velde \& W. Vandeweghe (éds) Sprachstruktur, Individuum und Gesellschaft, Tübingen, Niemeyer (103-112).

553 - (1981) «Problèmes de l'analyse automatique des prépositions du français ", in C. Schwarze (éd) Analyse des prépositions, Tübingen, Max Niemeyer Verlag (139-157).

554 - (1986) Die französische Präpositionalphrase und ihre automatische Analyse, Dudweiler, AQVerlag. 

insultes et autres études, Paris, Le Seuil (317340). [première publication in C. Hyart (éd) (1969) Mélanges Fohalle, Gembloux, Duculot (115-135)]

567 - (1984), « A propos de la distribution des complétives à temps fini et des compléments à l'infinitif en français ", Cahiers de Grammaire, 7 (74138).

ROBERGE, Y. (1998) «Les prépositions orphelines dans diverses variétés de français d'Amérique du Nord », in P. Brasseur (éd) Français d'Amérique : variation, créolisation, normalisation, Centre d'études canadiennes, Université d'Avignon (49-60).

ROBERGE, Y. \& ROSEN, N. (1999) « Preposition Stranding and que-Deletion in Varieties of North American French », Linguistica Atlantica, 21 (153-168).

ROEGIEST, E. (1977) «Problèmes de délimitation de la classe des prépositions », Revue des Langues Vivantes, 43 (419-434).

- (1981) «Grammaire des cas et sémantique des prépositions dans la construction verbale de quelques langues romanes ", Antwerp Papers in Linguistics, 23 (65-88).

ROHLFS, G. (1971) "Autour de l'accusatif prépositionnel dans les langues romanes ", Revue de linguistique romane, 35 (3123-34).

ROSIER, L. (2002) "Genre, le nuancier de sa grammaticalisation", Travaux de linguistique, 44 (79-88).

- (2005) «La polysémie des mots comme genre, style et type », in O. Soutet (éd) La polysémie, Paris, PUPS (231-243).

ROSSIGENSANE, N. (2001) «Pour une valeur aspectuelle des prépositions. Le cas des prépositions en emploi spatial dynamique », Syntaxe \& Sémantique, 3 (131-146).

ROTHEMBERG, M. (1974) Les verbes ä la fois transitifs et intransitifs en français contemporain, La Haye, Mouton.

ROULEAU, E. (2002) Est-ce "ä«, »de«, "par«, "pour«, "sur« ou »avec«? La préposition vue par un praticien, Québec, Linguatech.

ROUSSEAU, A. (1993) « Sur quelques conceptualisations de l'espace », Faits de langue, 1 (151-163).

RYCKMAN, T. \& GOTTFRIED, M. (1981) "Some Informational Properties of prepositions », Lingvisticae Investigationes, V: 1 (169-214).

SANTESSON, C. G. (1921) La particule 'cum' comme préposition dans les langues romanes, Upsal, Imprimerie Appelbergs Boktryckeri A. -B.

SARDA, L. (2005) «Fonctionnement des cadres spatiaux dans les résumés de film », Langue française, 148 (61-79).

SAUNIER, E. (1996), Identité lexicale et régulation de la variation sémantique. Contribution a l'étude des emplois de 'mettre', 'prendre', 'passer' et 'tenir', Thèse de doctorat de l'Université de Paris X-Nanterre.

SCALISE, S. (1969) «Verifica della funzionalità della 'teoria delle preposizioni' di Viggo Brandal », Lingua e Stile, 4 (139-159).

SCHMID, A. (1991) Mettre a toutes les sauces. Analyse sémanticosyntaxique des lexies complexes a base METTRE, Paris, Klincksieck. 
SCHOSLER, L. (éd) (2000), Le passif, Actes du colloque international organisé par l'Institut d'Etudes Romanes de l'Université de Copenhague, mars 1998, coll. Etudes romanes, 45.

SCHWARZE, C. (éd) (1981a) Analyse des prépositions, III ${ }^{\text {ème }}$ Colloque francoallemand de linguistique théorique du 2 au 4 février 1981 à Constance, Tübingen, Max Niemeyer Verlag.

- (1981b) «La préposition de la préposition", in C. Schwarze (éd) Analyse des prépositions, Tübingen, Max Niemeyer Verlag (215-223).

577 - (1992) «Farblose Präpositionen und Kasus im Französischen », in M.T. Schepping (éd) Zur Grammatik der Präpositionen, Konstanz, Universität Konstanz, Fachgruppe Sprachwissenschaft, Forschergruppe Lexikon, coll. Arbeitspapier, 51 (37-60).

578 - (1996) «Die farblosen Präpositionen des Französischen: vage Prädicate der Kasusmarker? », Romanische Forschungen, 108: 1/2 (1-22).

579 SCHEPPING, M.T. (1991), " The lexical meaning of the French preposition contre », in G. Rauh (éd) Approaches to Preposition, Tübingen, Gunter Narr Verlag (225-252). SEGUY, J. (1973) «L'accusatif prépositionnel en gascon et en français du sud-ouest », Travaux de linguistique et de littérature, $11: 1$ (429-433).

581 SELOSSE, P. (2002) "Pour une phytothérapie de la polysémie prépositionnelle ", Lingvisticae Investigationes, XXV : 2 (339-359).

SERAFIN, V. (1993) «Les circonstants en position finale. Problèmes d'incidence », in C. Guimier (éd) 1001 circonstants, Caen, Presses Universitaires de Caen (185-205).

SHEARS, F. (1922) Recherches sur les prépositions dans la prose du moyen français (XIV ${ }^{\text {ème }}$ et $\mathrm{X}^{\text {ème }}$ siècles), Paris, Honoré Champion. SHYLDKROT BATZEEV, H. (1987) «Les compléments de temps et de lieu sont-ils toujours des compléments circonstanciels? », Folia Linguistica, 21 :2/4 (229-247).

SLOBIN, D. I. (1996), « Two ways to travel : verbs of motion in English and Spanish ", in M. Shibatani \& \& S. A. Thompson (éds) Grammatical constructions : Their form and meaning, Oxford, Clarendon Press (195-220).

LOBIN, D. I. \& HOITING, N. (1994), « Reference to movement in spoken and signed languages : typological considerations », Proceedings of the Berkeley Linguistic Society 20 (487-505).

SORACE, A. (2000), "Gradients in Auxiliary Selection with Intransitive Verbs", Language, $76: 4$ (859-890).

SPANG-HANSSEN, E. (1963) Les prépositions incolores du français moderne, Copenhague, G.E.C., Gads Verlag.

589 - (1993) « De la structure des syntagmes à celle de l'espace. Essai sur les progrès réalisés dans l'étude des prépositions depuis une trentaine d'années », Langages, 110 (12-26).

590 - (2006) «Le rôle des prépositions spatiales dans la communication", in H. Nalke, I. Baron, H. Korzen, I. Korzen, H. H. Müller (éds) Grammatica. Festschrift in honour of Michael Herslund, Bern, Peter Lang AG (477-489).

SPANGHANSSEN, E. \& RUE, H. (1996) «The functions of locative prepositions », in E. EngbergPedersen et al. (éds) Content Expression and Structure, Studies in Danish 
Functional Grammar, Studies in Language Companion Series, Amsterdam/ Philapdelphia, John Benjamins (361-383). Mirail. nauki, 19 : 1 (71-77). (413-421). Linguistique, 27 : 2 (167-183). STÖCKLIN, J. (1971) « Problèmes de prépositions », Vox Romanica, 30 (89-97). STOSIC, D. (2002), «Par» et «à travers» dans l'expression des relations spatiales : comparaison entre le français et le serbo-croate, Thèse de l'Université de Toulouse-Le

TALMY, L. (1985), «Lexicalization patterns : semantic structure in lexical form », in T. Shopen (éd) Language typology and syntactic description, vol. 3, Cambridge, Cambridge University Press (57149). [repris et modifié dans 2000, tome 2 (21-146)]

- (1996), "Fictive motion in language and «ception»", in P. Bloom et al. (éds) Language and space, Cambridge, Mass., MIT Press. (211-276).

- (2000), Toward a cognitive semantics, 2 vol, Cambridge, Mass., MIT Press.

TAYALATI, F. (2005), Les adjectifs a complément au datif en français: Interface sémantique-syntaxe, Thèse de l'Université de Lille III - Charles de Gaulle.

TENCHEA, M. (1999) L'expression du temps dans le système des prépositions du français. Préposition et verbe, Timisoara, Editura Mirton.

TESNIÈRE, L. (1959), Eléments de syntaxe structurale, Paris, Klincksieck.

TERAVAKJAN, G. A. (1972) «Principy vydelenija sloznyx predlogov vo francuzskom jazyke (Distinction of complex prepositions in French) », Naucnye doklady Vyssej skoly, Filologiceskie nauki, $15: 1$ (90-97).

01 - (1977) «K probleme razgranicenija predloga I sojuza (On the differentiation of preposition and conjunction in French) ", Naucnye doklady Vyssej skoly, Filologiceskie

TOGEBY, K. (1964) « Prépositions latines et prépositions romanes », Lingua e Stile, 4

- (1984) Grammaire française, Volume IV: Les mots invariables, Copenhague, Akademisk Forlag, Université de Copenhague.

TOURATIER, C. (2001) « La notion de circonstant », Travaux, 17 (17-32).

TREMBLAY, M. (1996) "Lexical and nonlexical prepositions in French", in A.M. Di Sciullo (éd) Configurations, Somerville, Cascadilla Press (77-98).

- (1999) «Du statut des prépositions dans la grammaire», Revue Québécoise de

TREMBLAY, M. et al. (2003) « Les prépositions dans l'histoire du français : transitivité, grammaticalisation et lexicalisation ", Verbum, XXV : 4 (549-562).

TSUNEKAWA, K. et al. (2003) Nouveau Petit Royal. Dictionnaire japonais-français (2ème édition), Tokyo, Obunsha

TULLER, L. (1991) «Noms et prépositions à fonction adverbiale : une comparaison entre l'anglais, le français et le haoussa ", in C. Guimier (éd) L'adverbe dans tous ses états, Rennes, Presses Universitaires de Rennes, coll. Travaux du Cerlico, 4 (33-51).

TYLER, A. \& EVANS, V. (2003), The semantics of English prepositions : spatial scenes, embodied meaning and cognition, Cambridge, Cambridge University Press.

Société Polonaise de Linguistique, 31 (91-105). 
612 ULLMEREHRICH, V. (1981) «L'usage des prépositions indexicales dans un discours descriptif - La perspective déictique et la perspective inhérente », in C. Schwarze (éd) Analyse des prépositions, Tübingen, Max Niemeyer Verlag (224-250).

VAGUER, C. (2003), «La préposition dans: vecteur d'approximation? ", Revue de Sémantique et Pragmatique, 14 (135-155).

614 - (2006a) «Les prépositions: petits mots de grandes valeurs », Le Mensuel de l'Université - Premier média inter-universitaire pluridisciplinaire, Rubrique 'Etudes', $\mathrm{n}^{\circ} 16$, avril 2006.

615 - (2006b), «L'identité de la préposition dans : de l'identité à la coïncidence », Modèles Linguistiques, 53 (111-130).

616 VAGUER, C. \& LEEMAN, D. (2002) « Un étrange complément circonstanciel », Revue de la faculté des lettres en hommage à la mémoire de Maurice Gross, Fez (Maroc).

617 VALENTIN, P. (1970) «Les prépositions spatiales », Cahiers d'allemand (50-60).

618 VAN RAEMDONCK, D. (2001) « Adverbe et préposition : cousin, cousine ? ", Travaux de linguistique, 42/43 (59-70).

619 VANDELOISE, C. (éd) (1986) L'espace en français. Sémantique des prépositions spatiales, Paris, Editions du Seuil.

620 - (1993a) Langages n 110 : La couleur des prépositions, Paris, Larousse.

621 VANDELOISE, C. (1984) Description of Space in French, Thèse de doctorat, Université de Californie, San Diego, Publié sur Microfilm en 1986 par University Microfilms International, Ann Arbor, Michigan, USA.

622 - (1985a) La description linguistique de l'espace et du mouvement, Thèse de doctorat de l’École des Hautes Études en sciences sociales.

623 - (1985b) «Au-delà des descriptions géométriques et logiques de l'espace: une description fonctionnelle », Lingvisticae Investigationes, IX : 1 (109-129).

624 - (1986), L'espace en français : sémantique des prépositions spatiales, Paris, Le Seuil.

625 - (1987), « La préposition à et le principe d'anticipation », Langue française, 76 (77-111).

626 - (1990), «Les frontières entre les prépositions sur et à », Cahiers de Grammaire, 15 (159-184).

627 - (1991) Spatial prepositions. A Case Study from French, Chicago/London, The University of Chicago Press.

628 - (1992a) « Structure of Lexical Categories and Family Resemblances », LAUD, Duisburg.

629 - (1992b) «Quand le silence prend la parole », in L. Tasmowski \& A. ZribiHertz (éds) De la musique à la linguistique. Hommages à Nicolas Ruwet, Ghent, Blandijnberg 2, coll. Communications \& Cognition (634-639).

630 - (1993a) « Espace et motivation », Faits de langues, 1 (181-188).

631 - (1993b), «Les analyses de la préposition dans: faits linguistiques et effets méthodologiques », Lexique, 11 (15-40).

632 - (1994) «La catégorisation en linguistique cognitive », Le français moderne, $62: 1$ (79-98). 
633 - (2000) «Plus de clarté dans la relation porteur / porté », in M. Coene et al. (éds) Studia Lingvistica in honorem Liliane Tasmowski, Traiani Augusti Vestigia Pressa Sequamur, Padova, Unipress (753-769).

634 - (2003a) «Acquisition des termes spatiaux et relativisme linguistique », in C. Vandeloise (éd) Langues et cognition, Paris, Lavoisier (279-301).

635 - (2003b), "Les usages spatiaux de la préposition CONTRE », dans Recherches linguistiques, 26, 2003, «CONTRE Identité sémantique et variation catégorielle », P. Péroz éd., Université de Metz (15-44).

$636-(2005 a)$ « Family resemblances and the structure of spatial relationships », Corela, 3 : 2.

637 - (2005b), « Contre La rencontre atypique » dans Recherches linguistiques, 27, 2005, « Le mouvement dans la langue et la métalangue », P. Dendale éd., Université de Metz (121-150).

638 - (2006) « Are there spatial prepositions ? », in M. Hickmann \& S. Robert (éds) Space in Languages : Linguistic Systems and Cognitive Categories (137-154).

639 VAN GEENHOVEN, V. (2004), «For-adverbials, frequentative aspect, and pluractionality ", Natural Language Semantics, 12 (135-190).

640 VAN PETEGHEM, M. (à par.), «Le datif en français : un cas structural », in Mélanges linguistiques offerts à V. G. Gak, Moscou, Université Lomonossov.

641 VARGAS, C. (2001) "Compléments, circonstants et circonstanciels dans quelques grammaires universitaires du français », Travaux, 17 (51-72).

642 VAN VALIN, R. D. \& LAPOLLA, R. J. (1997), Syntax : structure, meaning and function, Cambridge, Cambridge University Press.

643 VASILIU, L. (1964) "Sur la définition de la préposition », Revue roumaine de linguistique, 9 (531-533).

644 - (1965) «La génération des déterminants nominaux prépositionnels », Revue roumaine de linguistique, 10 (275-279).

645 - (1967) «Some aspects of the grammar of verb-phrase with special reference to prepositional constructions », Revue roumaine de linguistique, 12 (341-367).

646 - (1968) «Some methodological remarks concerning a semantics of prepositions ", Revue roumaine de linguistique, 13 (541-549).

647 VERGUIN, J. (1967) « Prépositions, conjonctions, relatifs », Word, 23 (573-577).

648 VERNAY, H. (1974) Essai sur l'organisation de l'espace par divers systèmes linguistiques, München.

649 VICTORRI, B. (1999) « Le sens grammatical», Langages, 136 (85-105).

650 VIDALENC, J.L. (1986) «Prépositions : lexies ou cas?», in M. Verrac \& M. Pagnoux (éds) Lexique-Grammaire: Domaine Anglais, Saint-Étienne, Centre Interdisciplinaire d'Etude et de Recherches sur l'Expression Contemporaine (25-39).

651 VIEU, L. (1991) Sémantique des relations spatiales et inférences spatiotemporelles : une contribution à l'étude des structures formelles de l'espace en langage naturel, Thèse de doctorat de l'Université Paul Sabatier, Toulouse. 
VINET, M.T. \& BALDI, P. (1984) «Preposition Complementizers in French », Papers from the XII ${ }^{\text {th }}$ Linguistic Symposium on Romance Languages, Amsterdam, Benjamins (105-117).

VIVES, R. (1982) "Une analyse possible de certains compléments prépositionnels », Lingvisticae Investigationes, VI : 1 (227-233).

WAGNER, R. L. (1936) « Coordonnées spatiales et coordonnées temporelles », Revue de Linguistique Romane, 12 (144-164).

WEERENBECK, B. H. J. (1933) «L'infinitif prépositionnel sans sujet », Mélanges Salverda de Grave (389-398).

WEISSENBORN, J. (1981) «L'acquisition des prépositions spatiales : Problèmes cognitifs et linguistiques ", in C. Schwarze (éd) Analyse des prépositions, Tübingen, Max Niemeyer Verlag (251-285).

WHITTAKER, S. (2005) « Description syntaxique et discursive des syntagmes nominaux de type $\mathrm{N}$ dit + Complément adjectival, prépositionnel ou nominal », Journal of French Language Studies, $15: 1$ (83-96).

WIERZBICKA, A. (1993), « La quête des primitifs sémantiques », Langages, 98 (923).

WILMET, M. (1997), Grammaire critique du français, Paris, Duculot.

WITTGENSTEIN, L. (1986), Investigations philosophiques, Paris, Gallimard (1 ère éd. 1953).

WITTWER, J. \& GAUTE, J. (1974/1975) «La genèse des syntagmes dits compléments circonstanciels », Bulletin de Psychologie, 318 (844-856).

XATARD, V. (1989) Catégories grammaticales et distributions: les limites entre préposition, conjonction, adverbe, Thèse de doctorat nouveau régime, Université de Provence.

YAGUELLO, M. (1998) "Genre, une particule d'un genre nouveau ", Petits Faits de Langue, Paris, Le Seuil (18-24).

ZARING, L. (1991) «On prepositions and Casemarking in French », Revue canadienne de linguistique, $36: 4$ (363-378).

ZRIBI-HERTZ, A. (1984) «Prépositions orphelines et pronoms nuls », Recherches linguistiques de Vincennes, 12 (46-91).

- (1985) «Trou structural, catégorie vide, ellipse structurale, pronom nul : quatre concepts à préciser », Modèles linguistiques, $7: 1$ (131-148).

ZUBER, R. (1981) "Prépositions et intentionnalité », in C. Schwarze (éd) Analyse des prépositions, Tübingen, Max Niemeyer Verlag (286-295).

ZWICKY, A. M. (1987) «French prepositions: no peeking», Phonology Yearbook, 4 (211-227). 


\section{NOTES}

1. Liste des revues de langue française qui ont été "systématiquement dépouillées »: BULAG, Cahiers de grammaire, Cahiers de lexicologie, Cahiers de linguistique française, Cahiers de praxématique, Etude de linguistique appliquée, Faits de langues, Langages, Langue française, Le français moderne, Lexique, Lingvisticae Investigationes, Modèles linguistiques, Revue de sémantique et Pragmatique, Revue québécoise de linguistique, Semantikos, Sémiotiques, Syntaxe \& Sémantique, Travaux de linguistique, Travaux du Cerlico, Verbum...

\section{AUTEUR}

\section{CÉLINE VAGUER}

Université Paris X-Nanterre / CNRS UMR 7114 (MoDyCo) 